\title{
Integrating a Short Simulation Project into an InTroductory TransPort Phenomena Course
}

\author{
Jan Haelssig* and Devin O'Malley \\ Department of Process Engineering and Applied Science \\ Dalhousie University, Halifax, NS B3H 4R2 \\ *E-mail: Jan.Haelssig@dal.ca
}

\begin{abstract}
Transport phenomena is an integral part of many engineering curricula. Some programs, particularly in chemical engineering, teach transport phenomena in an integrated manner, but most teach heat, mass and momentum transfer in separate courses. Since powerful computers and software packages are now available to solve transport problems numerically, many current transport phenomena courses incorporate these tools for demonstration purposes. Many institutions also offer advanced level courses focusing on the use of this type of software, but the intuitive nature of modern software packages also permits the integration of these tools directly into introductory courses.

This paper summarizes efforts made in the Department of Process Engineering and Applied Science at Dalhousie University to integrate one such tool, COMSOL Multiphysics, directly into the introductory transport phenomena course. In an introductory course, the time available to study software tools is inherently limited, and therefore it is necessary to introduce these topics as efficiently as possible. In our course, several hands-on tutorials are used to introduce students to COMSOL. We avoid focusing too much on numerical methods, error estimation techniques, or even detailed model validation techniques. Instead, these tutorials focus on using commercial software to solve transport problems and demonstrating good modeling practices. Near the end of the course, students are asked to complete a short modeling project. In the latest iteration, students were asked to reproduce a simulation in a recent journal publication, to expand on the results, and comment on the significance of their findings. The main challenges related to implementing such a project include selecting problems that do not have unreasonably high computational requirements, the increased workload for teaching assistants, the required level of expertise among teaching assistants, and the increased workload for students.
\end{abstract}

Keywords: Transport Phenomena, Heat and Mass Transfer, COMSOL Multiphysics, Computational Fluid Dynamics (CFD).

\section{INTRODUCTION}

Dalhousie University's Department of Process Engineering and Applied Science offers accredited programs in chemical, environmental and materials engineering. After a review of the curricula for these programs, based partly on the new Canadian Engineering Accreditation Board's (CEAB) criteria for outcomes based assessments and partly on optimizing the delivery of these programs, an integrated transport phenomena course was added to the curriculum. This course replaced introductory heat and mass transfer courses in the chemical, environmental and materials engineering curricula. While developing this new integrated course, a particular effort was made to ensure that the applications discussed were relevant to all three of these disciplines. Additionally, in the hopes of modernizing the course content, several hands-on tutorials and a project were added to expose students to computational transport phenomena software. The software that has been used for the past two years is COMSOL Multiphysics. The tutorials, and specifically the project, give students the opportunity to learn a practical engineering tool and then to apply that tool to study a problem of their choice. Within the context of the CEAB's graduate attributes, a project like this helps to develop the students' investigation, computer tools, communication, and lifelong learning skills.

The purpose of this paper is to provide an overview of our experiences in incorporating computational software packages into our transport phenomena course. We hope that this information will allow other educators to apply similar techniques in comparable courses, while avoiding some potential pitfalls. A short literature review is included to justify why and how we have chosen to incorporate simulation software in this introductory course. The course structure and course projects from the fall of 2014 are then described. The results from these projects are analyzed from an educational perspective, and specific challenges are discussed. 


\section{LITERATURE REVIEW}

Over the past few decades there has been a dramatic increase in both the availability of powerful computational resources and advanced simulation software. The availability of these resources presents a great opportunity for engineers and scientists to solve problems of increasing complexity in both research and industry. However, with the increase in available computational tools, both traditional science and engineering programs must decide which types of tools to integrate into their curricula and how [1-4]. Engineering programs in particular usually already have a very heavy course load, and therefore there is usually little room to incorporate more core courses to teach specific software packages. Of course, it is possible and desirable to offer elective courses to permit students to gain deeper knowledge in a specific field, but such courses are unlikely to become part of the core of most programs.

For example, in chemical engineering, it is not unusual for schools to offer elective courses in Computational Fluid Dynamics (CFD) and/or multiphysics modeling [5-7], but it would be quite unusual for these to be part of the core curriculum. To provide all students with some exposure to such software tools, it is therefore necessary to incorporate them directly into some common core courses. This approach has been proposed by a number of authors in the chemical engineering education literature $[3,4]$. Also within chemical engineering there is a relatively long history of incorporating process simulation software directly into material and energy balance courses, and into process design courses. More recently, there has been an increased thrust to incorporate process simulation, dynamic process simulation and CFD software into unit operations lab, process control, instrumentation, reaction engineering and transport phenomena courses [see for example, 8-11].

When incorporating software into core engineering courses, it is important to keep in mind the specific goals that prompted the desire to do so in the first place. As discussed by Edgar [3], it is easy for faculty to lose sight of their students' needs and instead focus on the needs of their own research. There can also be a tendency to try to be too rigorous in the application of all aspects of the software or computational technique, which can overwhelm students with information. Although rigour is critical in research and professional practice, it is important to keep in mind that the learning objectives on first exposure to a software package are not normally to create practicing professionals. Instead, it is usually more beneficial to be very rigorous in the application of aspects that are specifically part of the learning objectives for the course, and to be less strict on other aspects.

\section{COURSE OVERVIEW}

\subsection{Structure, Objectives and Tutorials}

Transport phenomena courses are commonly taught using either a sequential approach or a unified approach, and there are many good textbooks available that fall into one of these categories. Courses structured using the sequential approach usually (but not always) begin by discussing momentum transport, and then move on to cover heat and mass transfer, while highlighting important differences and similarities between these phenomena. The unified approach introduces heat, mass and momentum transfer at the same time, but usually focuses on introducing one transport mechanism at a time. Regardless of the approach taken to introduce students to transport theory, it should be possible to introduce simulation software into courses following either of these two approaches in a similar way.

For the past three years at Dalhousie, we have followed a unified approach in our transport phenomena course. We first provide an introduction to different transport mechanisms. Diffusive steady-state transport with various applications is then introduced and analogies are drawn between the mechanisms. The conservation equations are then introduced and emphasis is placed on developing appropriate mathematical descriptions for various practical applications. Subsequently, convective transport is introduced, focusing primarily on relatively simple geometries and forced convection. Finally, transient diffusive transport is covered.

Simulation results and small demonstration experiments are sometimes shown during lectures and tutorials. The main objectives behind introducing simulation software in the course are: (a) to reinforce transport theory, (b) to reinforce proper modeling practice, (c) to allow students to visualize various transport mechanisms, and (d) to provide students with some foundational skills that they can use later in their studies and maybe even in their careers.

The course has many learning outcomes, focused mainly on developing a knowledge base for engineering and problem analysis skills. However, the course outcomes that are directly related to the use of simulation software are: (a) simplify and apply the differential and integral forms of the conservation equations for mass, momentum and energy to various physical, chemical and biological systems, (b) solve problems using the general balance equation with generation, convection and/or unsteady transport for various processes, (c) propose analytical solutions to the simplified transport equations, and (d) solve the transport equations numerically using commercial software for simple multi-dimensional, unsteady and coupled systems. 
Although there are many good commercial and opensource software packages available, we have chosen COMSOL Multiphysics as the software package to incorporate into the course. The primary reasons for this choice are that it provides a common user interface for pre-processing, solving and post-processing, and still has sufficient flexibility to be easily modified for unique situations. We have found that it takes students more time to become familiar with other software packages, for example ANSYS Fluent or OpenFOAM, and therefore these are probably more suited to dedicated courses.

In our course, COMSOL is normally introduced using three or four tutorials. In the fall of 2014, three formal tutorials were used, and one other simulation example was provided as a template for the project. The three formal tutorials were focused on simulating: (1) the transient development of the velocity profile in a fluid between two parallel plates, (2) two-dimensional heat conduction in a square rod, and (3) laminar and turbulent pipe flow.

The first tutorial introduces the COMSOL interface, the basic steps involved in developing a numerical model, the basic idea behind the finite element method, the importance of mesh independence studies, and the importance of model validation. It also reinforces the importance of boundary and initial conditions, introduces the transient solver, and covers basic post-processing techniques. The second tutorial focuses on steady-state heat conduction in a more complex geometry. It further reinforces the importance of a high quality mesh and model validation, introduces some more post-processing features, and introduces the steady-state solver. The third tutorial focuses on laminar and turbulent flow in pipes, specifically examining entrance lengths and local friction factor values. This tutorial introduces simulations involving axisymmetric geometries and the parametric studies feature of COMSOL. It also further reinforces good modeling practices and develops some more new post-processing skills.

\subsection{Course Projects}

The purpose of the course project was to allow the students to apply the COMSOL skills that they had obtained during the tutorials to a practical problem of their own interest. We hoped that the application of COMSOL to a larger engineering problem would expose the students to some of its more advanced features and thereby allow them to expand their skillset more rapidly. Our hope was that this exposure would permit the students to see how COMSOL (or similar software) could be used to help them to solve problems that they might encounter in the future. Of course, we knew that giving such a project would greatly increase our workload during the project because each group of students would encounter somewhat unique difficulties.
The course project required students to reproduce a simulation presented in a research article. They had the option to complete the project in pairs or individually. The deliverable was a conference style presentation (10 to 20 slides), following a specific template. The template closely followed the modeling strategy that had been employed during the tutorials. We also supplied a sample presentation for heat transfer from a tube bank in cross flow. The proposed structure of the presentation was: (i) problem description, (ii) geometry, (iii) model description, (iv) boundary conditions, (v) computational mesh, (vi) solver settings, (vii) validation, (viii) results and discussion, and (ix) summary and conclusions.

In the fall of 2014 we suggested eight projects, and students were also free to select their own project with the instructor's approval. The eight suggested projects are briefly described below.

Project 1 - Simulation of a simple shell-and-tube heat exchanger. In this case, the goal was to simulate heat transfer on the shell side of a simple, baffled shell-andtube heat exchanger $[13,14]$. The recommendation was to simulate a heat exchanger consisting of only five to seven tubes. A suggested study extension was to investigate how baffles and baffle spacing impacted the flow regime of the shell side fluid and ultimately the heat transfer efficiency.

Project 2 - Simulation of heat transfer in a simple coiled heat exchanger. For this project, students were asked to simulate heat transfer in a simple, coiled tube-intube heat exchanger [15-18]. The intention was to study convective heat transfer in a toroidal geometry. Several reference papers were provided to give the students some validation data and ideas for further study extensions.

Project 3 - Simulation of residence time distributions in water disinfection tanks. Maintaining an adequate residence time in water and wastewater disinfection systems is very important. The goal of this project was to estimate residence time distributions numerically for disinfection tanks with different geometries. As a basis, the study by Gualtieri [19] was provided.

Project 4 - Simulation of near-field toxic and/or flammable gas dispersion in simple geometries. The goal of this project was to simulate the downwind dispersion of pollutants and/or hazardous gases. The suggested geometry was something similar to a building with a stack and some other obstructions [20-22]. It was left up to the students whether or not they would do a fullscale or scaled-down analysis.

Project 5 - Simulation of phase change material (PCM) melting in two dimensions. Latent heat storage systems can be effective at storing thermal energy. This project explored the use of a cylinder full of paraffin wax that had hot water flowing through a pipe along the center to store energy. In order to optimize heat transfer, a number of fins extended from the pipe through the wax 
$[23,24]$. The suggestion was to first simulate the melting and then try to optimize the number of fins.

Project 6 - Simulation of high density polyethylene (HDPE) extrusion. The intention in this project was to simulate a simple polymer extrusion process. The objective was to simulate fluid flow and heat transfer in the extruding material. Since extrusion is a relatively complex process, a relatively simple case presented in the literature [25] was suggested. This case also included an analytical solution to permit validation.

Project 7 - Simulation of membrane evaporation. The purpose of this project was to develop a model to study the evaporation of water in a membrane contactor. As a basis for development of the model, we provided the students with the computational study by Shirazian and Ashrafizadeh [26]. The suggestion was that they could focus on geometry, air velocity and/or air humidity in their investigations.

Project 8 - Simulation of a distributor for a membrane cell. In this case, the objective was to investigate and optimize gas distribution within a membrane cell [27]. It was suggested that the students should replicate the membrane cell geometry provided in the journal article, and then to study some other geometrical effects.

\section{RESULTS AND DISCUSSIONS}

\subsection{Analysis of Student Project Submissions}

The suggested course projects were not all equally popular. As shown in Table 1, Projects 6 and 8 were not selected by any students. The main reason for this may have been that they felt uncomfortable with nonNewtonian fluids (Project 6) and did not want to draw complex geometries (Project 8). Additionally, 21 out of the 92 students who ultimately completed the course successfully did not complete the course project. The course project was only worth $5 \%$ of the course mark, and it appears that these students thought that their time could be better spent on other tasks.

Table 1: Summary of Student Project Choices (Project 6 and 8 were not selected).

\begin{tabular}{lc}
\hline & Number of Students \\
\hline Project 1 & 16 \\
Project 2 & 6 \\
Project 3 & 22 \\
Project 4 & 8 \\
Project 5 & 9 \\
Project 7 & 2 \\
Other & 8 \\
Incomplete & 21 \\
\hline
\end{tabular}

Out of the projects that were completed by the students, the largest number chose to complete projects 1 and 3. We are not entirely sure why students preferred certain projects over others, but part of the reason may have been that a large number of the students were concurrently enrolled in a thermal unit operations course (closely related to projects 1 and 2) and a reaction engineering course (with material on residence time distributions being related to Project 3). Additionally, the materials engineering students in the class seemed to prefer Project 5, and environmental engineering students with interests in air pollution seemed to prefer Project 4. Eight other students decided to choose projects based on their own specific interests.

Overall, we were quite impressed with the quality of the simulations that were submitted. Students generally adhered to the suggested modeling methodology (see section 3.3 above) quite well. However, only a relatively small subset of students went above the basic requirement of implementing a simulation presented in the journal publication. The reason why most students decided not to pursue a large number of study extensions is likely because only three weeks were given to complete the project, and the weighting toward the overall course mark was relatively low. Nonetheless, many students were quite passionate about the project, and it certainly seemed to spark future interests in some individuals. Examples of a portion of submissions for projects 1 and 3 (unaltered except for combining graphics) are provided in Figs. 1 and 2.

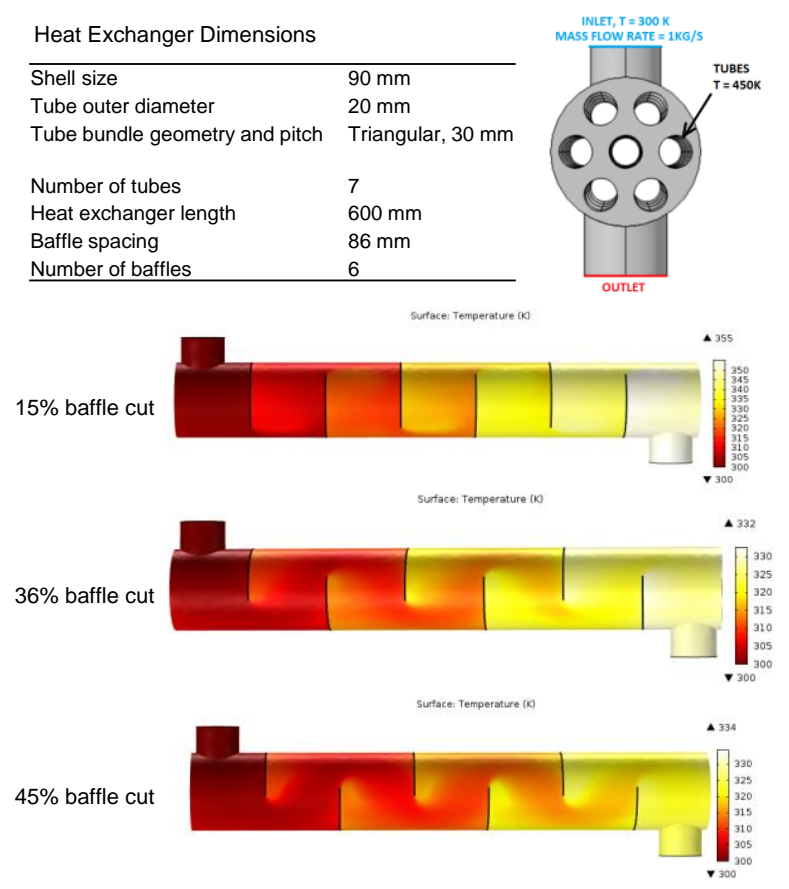

Fig. 1. Example of student submission showing a heat exchanger geometry and optimization study for Project 1 . 


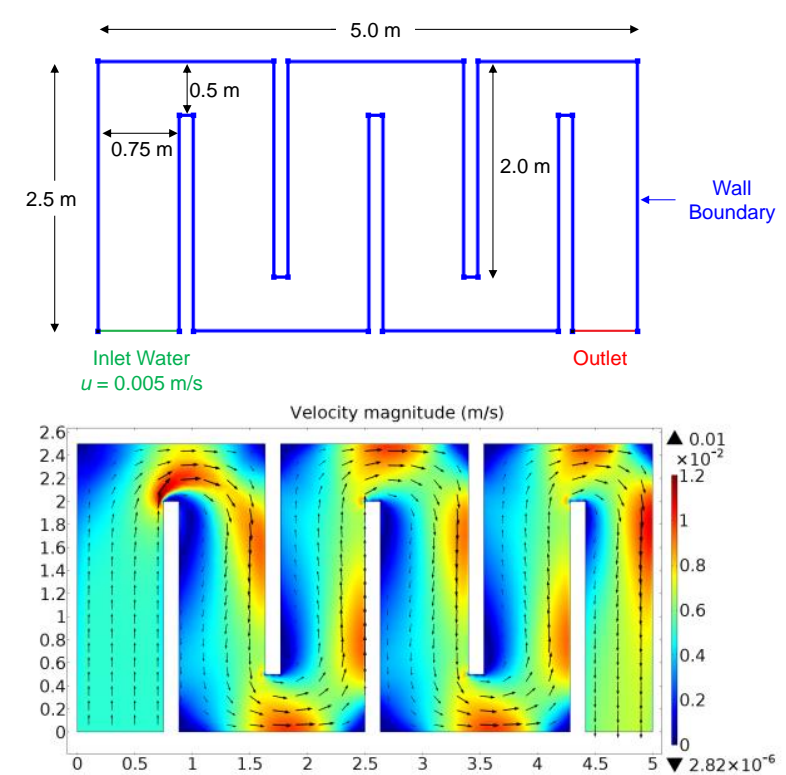

Fig. 2. Example of student submission showing the geometry and velocity profile for Project 3 .

\subsection{Student Feedback and Analysis}

Unfortunately, during the fall semester of 2014, we did not ask the students to complete a comprehensive survey to reflect on the course project. We therefore only have a limited amount of quantitative results, and have to rely primarily on the Student Ratings of Instruction (SRI) and other anecdotal evidence. In 2013 we first began to introduce COMSOL into our transport phenomena course, and the general feedback from the students was that they thought that it was an interesting and useful tool, but that they would have liked to have had more time to explore its features. This feedback, combined with the fact that we wanted to allow students in each our three programs taking the course to explore more relevant problems to their own interests, was the main reason that we decided to introduce a simulation project in the first place.

Since introducing COMSOL into this course in the fall of 2013 we have seen a continuous increase in the number of students wanting to use COMSOL during their senior design projects and in research projects. Over the previous year, there were only positive changes in the SRIs for the course in the fall of 2014. This seems to indicate that, even if the students thought that the course project was a lot of work, they seemed to appreciate the course content. The biggest increase in the SRIs was for "instructor enthusiasm", which is probably directly correlated with the instructor's interests in CFD.

On the SRIs, most students (4.13/5) indicated that they will continue to try to apply concepts learned in the course to other situations. A significant portion (3.73/5) indicated that the introduction to the use of COMSOL to solve complex transport problems was a useful part of the course, and that they could see how such engineering software could be applied to other problems. Most students (4.16/5) indicated that they felt that they achieved the learning "outcomes" listed in the course syllabus.

The only negative comments about the use of COMSOL in the course were about to the increased workload related to the course project, and frustration because some students felt unprepared for the level of difficulty of the project. We are somewhat unapologetic about the latter frustrations because we feel that most learning occurs when struggling through a truly openended and challenging problem. However, we are concerned about the workload problem, especially because the project was completed at the end of a busy term. In future years we will therefore follow one of our students' enlightened suggestions and give the project right at the beginning of the semester. We will then have a series of deliverables related to the project due at various stages throughout the semester.

\section{CONCLUSIONS AND FUTURE WORK}

This paper presented an overview of our experiences in incorporating COMSOL Multiphysics into a transport phenomena course. The course introduces COMSOL through a sequence of three to four tutorials, and then students are required to complete a simulation project. The project forced students to focus on key modeling areas as part of the project deliverable. Based on the project submissions, it is clear that those who completed the project tended to do quite well. Therefore, those who completed the project seemed to successfully meet the desired learning objectives.

From the feedback it is clear that students seem enthusiastic to learn simulation software and see the benefit in solving future problems, but they can be frustrated with time constraints. The level of frustration seems to increase even more when the tasks are very open-ended. In future years we will mitigate this problem by giving the project earlier and setting a series of due dates for various deliverables related to the project.

We should also note that the course project placed a significant additional workload on the instructor and teaching assistant. Most of this additional time was spent on resolving model and software related issues (i.e., troubleshooting). We would therefore only suggest the incorporation of such a project into a course if the instructor(s) are very comfortable with the chosen software package.

\section{Acknowledgements}

Financial support from Dalhousie's Faculty of Engineering for the educational initiatives presented in 
this paper is gratefully acknowledged. We would also like to thank the students of PEAS 3600 from fall 2013 and 2014 for their participation and useful feedback.

\section{References}

[1] Rubin H. Landau, "Computational Physics Education; why, what and how," Computer Physics Communications, no. 177, pp. 191-194, February 2012.

[2] W. Wiechert, "The role of modeling in computational science education," Future Generation Computer System, no. 19, pp. 1363-1374, 2003.

[3] Thomas F. Edgar, "Enhancing the Undergraduate Computing Experience," Chemical Engineering Education, pp. 231-238, Summer 2006.

[4] Mordechai Shacham, Michael B. Cutlip, and Neima Brauner, "From Numerical Problem Solving to ModelBased Experimentation - Incorporating Computer-Based Tools of Various Scales Into the ChE Curriculum" Chemical Engineering Education, pp. 315-321, Fall 2009.

[5] Desmond Adair, Zhumabay Bakenov, and Martin Jaeger, "Building on a traditional chemical engineering curriculum using computational fluid dynamics," Education for Chemical Engineers, no. 9, pp. 85-93, 2014.

[6] V. V. R. Kaushik, S. Ghosh, G. Das, and P. K. Das, "CFD Modeling of water flow through sudden contraction and expansion in a horizontal pipe," Chemical Engineering Education, pp. 30-36, Winter 2011.

[7] Estanislao Ortiz-Rodriguez, Jorge Vazquez-Arenas, and Luis A. Ricardez-Sandoval, "An undergraduate course in modeling and simulation of multiphysics systems," Chemical Engineering Education, vol. 44, no. 4, pp. 299305, Fall 2010.

[8] Tiina M. Komulainen, Rasmus Enemark-Rasmussen, Gürkan Sin, John P. Fletcher and David Cameron, "Experiences on dynamic simulation software in chemical engineering education," Education for Chemical Engineers, no. 7, pp. 153-162, 2012.

[9] Benjamin J. Lawrence, Jason D. Beene, Sundararajan V. Madihally, and Randy S. Lewis, "Incorporating nonideal reactors in a junior-level course: using computational fluid dynamics (CFD)," Chemical Engineering Education, pp. 136-141, 2004.

[10] Luis M. Madeira, Manuel A. Alves, and Alírio E. Rodrigues, "Teaching nonideal reactors with CFD tools," Chemical Engineering Education, pp. 154-160, 2004.

[11] William M. Clark, Yaminah Z. Jackson, Michael T. Morin, and Giacomo P. Ferraro, "Combining Experiments and Simulation of Gas Absorption for Teaching Mass
Transfer Fundamentals: Removing $\mathrm{CO}_{2}$ from air using water and $\mathrm{NaOH}$," Chemical Engineering Education, vol. 45, no. 2, pp. 133-143, 2011.

[13] Ender Ozden and Ilker Tari, "Shell side CFD analysis of a small shell-and-tube heat exchanger," Energy Conversion and Management, no. 51, pp. 1004-1014, 2010.

[14] K. S. Arjun and K. B. Gopu, "Design of Shell-and-Tube Heat Exchanger Using Computational Fluid Dynamics Tools," Research Journal of Engineering Sciences, vol. 3, no. 7, pp. 8-16, 2014.

[15] Luca Cattani, "Numerical Investigation of The Convective Heat Transfer Enhancement in Coil Tubed," in Proc. of the 2012 COMSOL Conference (Milan), 2012.

[16] J. S. Jayakumar, S. M. Mahajani, J. C. Mandal, P. K. Vijayan, and Rhoidas Bhoi, "Experimental and CFD estimation of heat transfer in helically coiled heat exchangers," Chemical Engineering Research and Design, no. 86, pp. 221-232, 2008.

[17] Vimal Kumar, Supreet Saini, Manish Sharma, and K. D. P Nigam, "Pressure drop and heat transfer study in tube-intube helical heat exchanger," Chemical Engineering Science, no. 61, pp. 4403-4416, 2006.

[18] Vimal Kumar, Burhanuddin Faizee, Monisha Mridha, and K. D. P Nigam, "Numerical studies of a tube-in-tube helically coiled heat exchanger," Chemical Engineering and Processing, no. 47, pp. 2287-2295, 2008.

[19] C. Gualtieri, "Numerical Simulation of RTD in Contact Tanks with Comsol Multiphysics 3.2b," Available as of April 19, 2015 from www.comsol.com/paper/download/8141/Gualtieri.pdf

[20] Mauricio Chavez, Bodhisatta Hajra, Ted Stathopoulos, and Ali Bahloul, "Near-field pollutant dispersion in the built environment by CFD and wind tunnel simulations," J. Wind Eng. Ind. Aerodyn., no. 99, pp. 330-339, 2011.

[21] P. Gousseau, B. Blocken, and G. J. F. van Heijst, "CFD simulation of pollutant dispersion around isolated buildings: On the role of convective and turbulent mass fluxes in the prediction accuracy," Journal of Hazardous Materials, no. 194, pp. 422-434, 2011.

[22] J. D. McAlpine and Michael Ruby, "Using CFD to Study Air Quality in Urban Microenvironments," Environmental Sciences and Environmental Computing, vol. 2, pp. 1-31, 2004.

[23] Dominic Groulx and Wilson Ogoh, "Solid-Liquid Phase Change Simulation Applied to a Cylindrical Latent Heat Storage System," in Proc. COMSOL Conference (Boston), 2009.

[24] Wilson Ogoh and Dominic Groulx, "Effects of the heat transfer fluid velocity on the storage characteristics of a 
cylindrical latent heat energy storage system: a numerical study," Heat Mass Transfer, no. 48, pp. 439-449, 2012.

[25] A. G. Mamalis, K. N. Spentzas, G. Kouzilos, I. Theodorakopoulos, and N. G. Pantelelis, "On the HighDensity Polyethylene Extrusion: Numerical, Analytical and Experimental Modeling," Advances in Polymer Technology, vol. 29, no. 3, pp. 173-184, 2010.

[26] Saeed Shirazian and Seyed N. Ashrafizadeh, “3D Modeling and Simulation of Mass Transfer in Vapor
Transport through Porous Membranes," Chemical Engineering \& Technology, vol. 36, no. 1, pp. 177-185, 2013.

[27] Nikhil Kawachale, Deepak M. Kirpalani, and Ashwani Kumar, "A mass transport and hydrodynamic evaluation of membrane separation cell," Chemical Engineering and Processing, vol. 49, pp. 680-688, 2010. 